\title{
Hierarchical Network Management Based on Extended SNMP
}

\author{
Member Yu Guo (Yamagata University) \\ Non-member Takao Akatsuka (Yamagata University) \\ Non-member Yukio Hiranaka (Yamagata University)
}

In this paper, we present an analysis of the problems involved in both centralized and distributed network management architectures and present a scheme of Hierarchical Network Management based on Extended SNMP as our solution. The status field in SNMP PDU Format is newly defined so that various management policies can be encapsulated into the SNMP PDU and sent from main manager to sub manager. The management task, which achieves concrete management activities on side of the sub manage, is created and executed according to management policy. One of the advantages of the proposed method is distribution of all monitoring tasks and data processing tasks to each sub manager so that the load of main manager and the traffic of management information on the backbone network can be significantly decreased. In addition, it is possible to obtain high-level management result from the sub manager directly by using free-designed management policy. The management framework is easy to implement on all versions of SNMP and makes the management system more flexible and scalable. Implementation examples are also given.

Keywords: management policy, management task, main/sub manager, SNMP, PDU, MIB

\section{Introduction}

Network Management is the process of monitoring and maintaining a complex network so as to maximizes its efficiency and productivity. As networks have become increasingly complex, both logically and geographically, network management has become more challenging.

In most network management, centralized management architecture is well-used ${ }^{(1)}$. In this architecture, a central management station, on which the management application is being run, gathers the monitoring data from the managed devices through the network and then makes a decision and transfers the maintenance data to the devices by the same method. Since the central management station must communicate with the most of the end devices, the valuable bandwidth of the network backbone will be occupied by management information traffic, especially in remote network monitoring which must be implemented via the WAN links. Several distributed management approaches have been proposed for solving these problems. One is M2M (Manager-to-Manager) MIB that is developed within SNMPv2 in order to distribute controlling and monitoring functions of network management among multiple management stations and to coordinate those stations ${ }^{(2)}$.
Another very popular solution, known as RMON (Remote Network Monitoring), is to place remote management devices (RMON Probes) on different remote segments. The RMON Probe, which has been the important complementary function of SNMP-based Network Management so far, can monitor the remote network and efficiently provide management station with statistical information ${ }^{(3)(4)}$. On the other hand, some commercially available network management software applications have recently been shown to be capable of management distribution as well. An example of this is a Distributed Management Console, which is usually authorized to perform special management functions such as network performance or configuration management or to manage one specified network. Another example of commercial software is the Management Information Collection Station, which always monitors its local network and only forwards valuable management information to management station.

However, there are some deficiencies associated with the above network management solutions. The main deficiencies are as follows: (1) Objects in both M2M MIB and RMON MIB have been defined in advance so that the function of management is restricted within narrow selections. What those methods could do is only to monitor 
and record the value of characteristic variables of MIB, check some thresholds, and send alarm notifications to the main management station. Indeed, there is no flexibility and scalability to make adaptations to management necessary for a rapidly developing network. (2) When monitoring a remote network, only the traffic pattern of every sub network or segment is listened to and captured. It is impossible to obtain detailed management information from any of the devices in a remote network by the RMON method. The same problem occurs when managing remote VLANs (Virtual LAN), which has mobility and is considered as an essential switched LAN environment in today's enterprise network. (3) The agent with M2M and the RMON probe cannot perform a true proxy role for the main management station. Some high-level results of management such as average TCP packet loss rates of a certain network device, average traffic on a VLAN, or status checks of specified network interfaces that need a series of computation processes or logical operations, could not be obtained directly by using those methods. (4) The Distributed Management Console still resembles a centralized management station because the management structure has not been improved, except using several computers instead of the original management station. The Management Information Collection Station behaves in exactly the same manner as a filter machine that chooses valuable MIB information for the management station. Furthermore, in any case, it could not obtain the high-level results of management. In summary, the management function of current distributed management method is limited to what we have described above. Management topology always keeps a fixed structure in three levels. The main management station also has the same problems occurring appearing in the centralized management method when processing the high-level management results.

In this paper, we present a scheme, which we call SNMP-based Hierarchical Network Management. The purpose is to develop a flexible and scalable management method to manage large-scale distributed networks efficiently. We use the developed SNMP PDU (Protocol Data Unit) to transfer management policy to the sub manager. The management policy, which consists of a set of operations, creates management task for achieving a specific management function service required by the main manager. Several different management tasks can be run at the same time on one sub manager to monitor the sub network and to process the management information with the SNMP or RMON method in accordance with the specified start time and execution interval. A great variety of operator and operand combinations can achieve various goals of management as we expect. Our approach can also supply a platform for the development of any hierarchical management applications. This paper is organized as follows: In Section 2, we describe the framework of hierarchical management system. The SNMP PDU packet is analyzed and enhanced to carry management policy in Section 3. In Section 4, we discuss how to store the management policy on the sub manager side; and in Section 5 , how to implement our method under TCP/IP network environment and implementation examples in several cases are shown. Our conclusions are given in Section 6.

\section{Hierarchical Network Management Framework}

Since we know that more and more network devices are being connected to networks, obviously it is not an appropriate solution to use a single management station to monitor an entire network. On the other hand, because of some limited management functions, the distributed management method is not the best choice in large-scale complex network management either. As a solution to these problems, the Hierarchical Network Management method as shown in Fig. 1 has been developed.

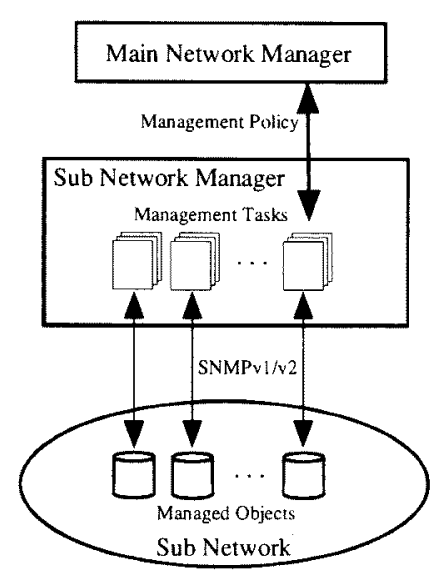

Fig. 1. The Framework of Hierarchical Network Management

The basic view of this method is that the main management station (main manager) assigns the management policy only to the mid-level manager (sub manager) and then waits for results sent from the sub manager. Thus the main manager can avoid communicating with the low-level or remote network devices directly, and the bandwidth of the backbone network and resources of the main management station can be saved. A new mid-level 
sub manager can be inserted between the main manager and current mid-level sub manager or between the current midlevel sub manager and the low-level sub network. It is also possible for the sub manager that serves as network manager for its sub network to create the management tasks in accordance with the management policies sent from the main manager. The management task can use SNMP to access both normal MIB II and RMON MIB maintained by not only the sub manager itself but also other devices including RMON probes which can be placed in the lowestlevel sub networks if necessary. Some calculated results can also be obtained by the management task as well.

The management policy consists of a queue of operations. Each operation includes an operator that represents the management command and an operand that represents the managed object, which also includes IP address and object identifier. Fig. 2 gives the structure of management policy.

\begin{tabular}{|l|l|}
\hline Network Management Policy \\
\hline Operation1 & Operation2 \\
\hline Operator & Operand \\
\hline
\end{tabular}

Fig. 2. The Structure of Network Management Policy

The principal procedure of hierarchical management operations may be described as following:

(a) The main manager assembles a new management policy and assigns it to the sub manager.

(b) The sub manager stores the management policy including an operator table and an operand table into the MPMIB (management policy MIB).

(c) The sub manager creates and executes the management task by reading the contents of the MPMIB. The processed result is saved into the MRMIB (management result MIB), which can be further shared by the main manager. The management policy stored in the MPMIB can be executed periodically or any times requested by the main manager.

(d) The results in the MRMIB are either transferred to the main manager by SNMP Trap PDU or retrieved by the main manager by using GetRequest/GetNextRequest.

\section{Extended SNMP PDU}

How to take advantage of SNMP to realize the Hierarchical Network Management is an important theme in our approach, since the SNMP is simply implemented within almost every end-to-end internetworking device and acts as a basic tool for current network management ${ }^{(5)}$. SNMP is based on the manager/agent model, and its agent requires minimal software. Many vendors of both agent and management systems implement SNMP, and continue to enhance it ${ }^{(6)}$. From the standpoint of interoperability, SNMP-based implementation is a sensible choice. At the same time, the security issue in our network management system, including authentication, access control, etc., can be derived from SNMP. Utilization of SNMP in our solution includes encapsulating management policies into the SNMP PDU, mapping them to the sub manager, and using the mechanism of MIB to store and manage management policies, management information and operation results. We focus on the above issues in the following subsections.

\subsection{Error-Status \& Error-Index Field of SNMP PDU}

SNMP itself has been frequently enhanced because of its widespread usage. The current SNMPv2, which was upgraded from its version 1, gives two new PDU definitions, namely GetBulkRequest PDU to effectively obtain large data block, and InformRequest PDU to implement Trap transmission between two managers. Some procedures for the creation and deletion of the MIB table row are also defined in SNMPv2 for supporting RMON. The upcoming SNMPv3 reuses the SNMPv2 Draft Standard documents and is essentially SNMPv2 plus security and administration functions.

Nevertheless, all upgraded versions of SNMP inherit the same basic structure and components from their earlier form ${ }^{(7)}$. According to the IETF documents RFC1157 ${ }^{(8)}$, RFC1448 (9), RFC1908 (10) and RFC2273 (11), the SMNPv2/SNMPv3 PDU also keeps the same basic structure of the PDU format as SNMPv1 PDU, especially the PDU format of GetRequest, GetNextRequest, SetRequest, GetBulkRequest, and InformRequest as shown in Fig. 3. From the same IETF documents, we have also found that the SNMP agent ignores the values in both the Error-Status field and the Error-Index field when the agent receives the above SNMP PDUs except in the case of GetBulkRequest PDU. The GetBulkRequest PDU (PDU Type $=5$ ) uses above two fields to indicate the values of Non-Repeaters and Max-Repetitions, although the GetBulkRequest PDU has the same PDU structure of other SNMP PDU as shown in Fig. 3. In our solution, we redefine these two fields for the purpose of assigning to the prior SNMP PDU some new functions to transfer management 
policy from main manager to sub manager under the hierarchical network management environment. We refer to these modified PDUs as Extended SNMP PDU, including GetRequest (PDU Type=0), GetNextRequest (PDU Type=1), SetRequest (PDU Type=3), and InformRequest (PDU Type=6). On the other hand, a software component can be inserted between the network daemon program and the SNMP agent daemon program for purpose of analyzing the PDU Type and the values of the Error-Status and the Error-Index field. As a result, the sub manager can distinguish the Extended SNMP PDU from other normal type of SNMP PDU it has received.

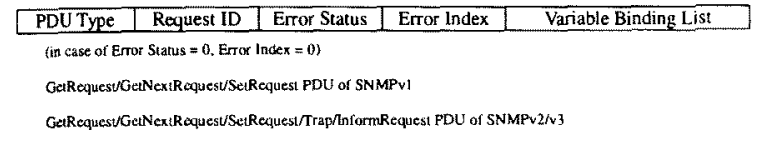

Fig. 3. SNMPv1/v2/v3 PDU Structure (from Manager to Agent)

Incidentally, according to the definition of ASN.1 (Abstract Syntax Notation One) ${ }^{(12)}$, the PDU Type field does not actually exist in either SNMPv1 PDU or SNMPv2 PDU. It will be created and come into view as a by-product automatically when any SNMP PDU is encoded by BER (Basic Encoding Rules) before it is encapsulated into the UDP packet ${ }^{(5)(13)(15)}$. But this has no influence on identifying the specified SNMP PDU on the side of the sub manager.

3.2 SNMP PDU Developing According to the Hierarchical Network Management Framework as described in Section 2, the management policy consisting of a queue of operator-operand pairs must be sent to the sub manager. In order to identify that the current PDU is an Extended SNMP PDU in which the management policy is encapsulated, a special mark needs to be assigned to each Extended SNMP PDU. We insert two identifiers, $X$ octet and $Y$ octet, into reusable Error-Status field and Error-Index field within SNMP PDU to tell the sub manager what it should do and how to do it. At the same time, a string of arguments is inserted into the Variable-Binding List located on the rear of the same SNMP PDU. As shown in Fig. 4, if the $\mathrm{XY}=\# F F F F$, it means that the next Variable-Binding List to arrive combined in the same SNMP PDU is part of the operators of management policy. The part of the operator made up of a string of opCode (operating code) tells the sub manager how to create the management task, and what kind of management operation it should perform. If the $\# 00<\mathrm{XY}<\# F F F F$, it means that the next VariableBinding List to arrive is a string of operands which stands for the operated objects of opCode. The value of XY indicates which management policy the operand belongs to. This case is illustrated in Fig. 5.

In order to make the same policy (operator) work with different operands, we use different Extended SNMP PDUs to transfer opCode and operands. If the same policy were to be downloaded to the same sub manager again, the main manager could send the identifier of the same policy and new operands to the sub manager. Note that the policyExecStatus and operandExecStatus, in Fig. 4 and Fig. 5 , are two variables, not MIB objects. They are used with $X Y$ to inform the sub manager if it starts to create the management task as soon as it receives current operator or operand Extended SNMP PDU. If yes, the sub manager retrieves the policy (operator) according to the policy identifier from MPMIB. We will describe this again in the next section. As a result, the efficiency of transferring between main manager and sub manager can obviously be improved in the above frequently occurring case.

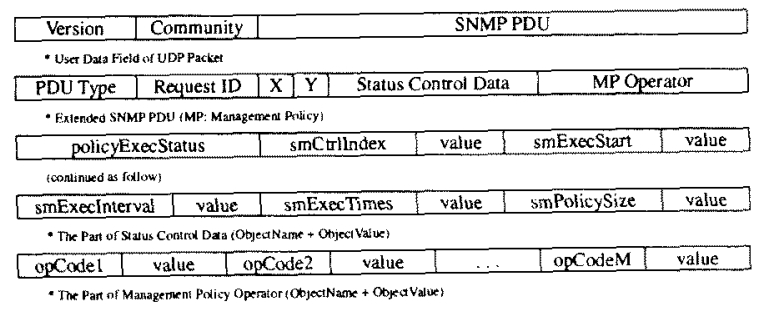

Fig. 4. Extended SNMP PDU (when $X Y=\# F F F F$ ) for Operator of Management Policy

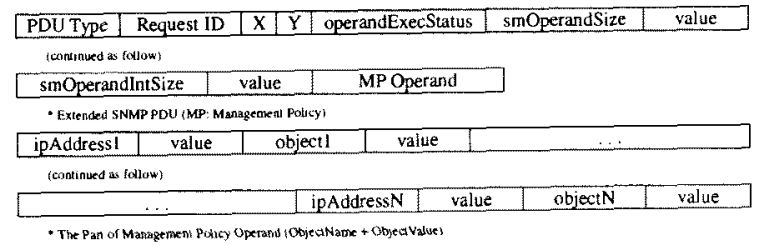

Fig. 5. Extended SNMP PDU (when \#00< XY < \#FFFF) for Operand of Management Policy

In addition, the main manager can retrieve the result data from the MIB on a sub manager by sending GetRequest or GetNextRequest PDU, or it can delete the result record when it is no longer considered valuable by sending SetRequest PDU.

\section{Designing MPMIB}

The sub manager can run different management tasks in accordance with several different management policies 
simultaneously. Another issue to be resolved is the determination of procedures for saving, managing and operating a number of management policies on the sub manager. One possibility is to store management policies in the form of MIB, since the main manager frequently uses SetRequest PDU to send them to the sub manager. We define a new MIB for sub manager, called MPMIB and put the management policy into it. The MPMIB operation that inserts or deletes a row also follows the operation procedure for normal MIB. Similarly, the MRMIB used to store the management result can be configured in the same way.

4.1 Structure of MPMIB As we stated in Section 3.2, operator and operand of management policy are sent to a sub manager by different Extended SNMP PDUs. Therefore, we can put the operator and operand into different tables within one MPMIB by using SEQUENCE type and SEQUENCE OF type of ASN.1.

In the Operator Table of MPMIB, the smCtrlIndex object is a unique operating identifier (\#0001- \#EFFF) created in turn by the main manager to distinguish the management policy from others. In other words, it defines each row of the table. The smPolicySize object means the total number of valid opCode in the management policy. Moreover, both smCtrlIndex and smPolicySize objects are used as INDEX object to specify instances for columnar objects within each row that comprises the table. Further information about INDEX object can be found in RFC1212 ${ }^{(13)}$. In addition, execution start time, execution interval, and execution times of the management policy are indicated by smExecStart, smExecInterval, and smEcexTimes object. The queue of opCode indicating the various operators becomes a part of the columns in the table. In the Operand Table of MPMIB, the value of smOperandIndex object is equal to the value of $\mathrm{XY}$, which was assigned from the value of smCtrlIndex object by the main manager. The smoperandIndex object refers to the entry index of the Operand Table for the management policy. The smoperandSize object refers to the number of operand. The combination of smOperandIndex object and smOperandSize object is also used as an INDEX object. Every operand consists of a physical IP address and a MIB object identifier of the managed object. The smOperandIntSize object refers to the number of special operands that have an IP address of 000.000 .000 .000 . The definition of MPMIB is given in Table. 1.

The two row entries, one indicating the management policy in the operator table and the other one indicating the operand in the operand table, are associated with one another by a unique identifier which is the value of smCtrlIndex object. Therefore, any of the management policies can be executed again with either the same operand or with the other operand newly assigned with the same value of smCtrlIndex by the main manager. Since we know that two variables, policyExecStatus and operandExecStatus, are used with XY to inform the sub manager if it starts to create the management task immediately after receiving either operator or operand Extended SNMP PDU. If yes, the sub manager retrieves the policy (operator) according to the policy identifier from MPMIB. Both $M$ and $N$ should be defined within \#01-\#FF(1-255) to keep the size of MPMIB within a limited scope.

Table 1. MPMIB Object Definition

\begin{tabular}{|c|c|c|}
\hline Object Tables & MPMIB Objects & SYNTAX in SMI \\
\hline \multirow{9}{*}{$\begin{array}{l}\text { smOperator } \\
\text { table }\end{array}$} & smCtrllndex & INTEGER (1..65534) \\
\hline & smExecStart & INTEGER (0..65535) \\
\hline & smExecInterval & INTEGER $(0 . .65535)$ \\
\hline & smPolicyTimes & INTEGER $(0.65535)$ \\
\hline & smPolicySize & INTEGER $(1.255)$ \\
\hline & \begin{tabular}{|l|} 
opCode 1 \\
\end{tabular} & OCTET STRING \\
\hline & opCode 2 & OCTET STRING \\
\hline & ; & $:$ \\
\hline & opCodeM & OCTET STRING \\
\hline \multirow{10}{*}{$\begin{array}{l}\text { smOperand } \\
\text { table }\end{array}$} & smOperandIndex & INTEGER (1..65534) \\
\hline & smOperandSize & INTEGER (1.255) \\
\hline & smOperandIntSize & INTEGER (1..255) \\
\hline & ipAddress 1 & IpAddress \\
\hline & object 1 & OCTET STRING \\
\hline & ipAddress 2 & IpAddress \\
\hline & object 2 & OCTET STRING \\
\hline & $:$ & $:$ \\
\hline & ipAddress $\mathrm{N}$ & IpAddress \\
\hline & objectN & OCTET STRING \\
\hline
\end{tabular}

4.2 Structure of MRMIB The MRMIB is used to save the management result after the sub manager executes the management task. It can be modified and retrieved by both main manager and sub manager. In order to deal with GetRequest / GetNextRequest / GetBulkRequest / InformRequest / SetRequest easily, the MRMIB also takes the same form as the normal MIB.

A Result Table exists in the MRMIB. In this table, the smResultIndex object, which is assigned to the same value as the smCrtlindex, refers to the management result created by the management policy. This object is also a retrieval key to help the main manager or sub manager search the history value of the result if it still exists. The smResult Time refers to the time when the result was created. The number of result data, maximum to \#FF(255), is stored in smResultSize object, and the result data itself is stored in smResultData object. 


\section{Implementation on TCP/IP Network}

To implement the hierarchical network management system on a real TCP/IP network, having the sub manager based on SNMPv2 is recommended, since several agents on different ports of one machine can be run at the same time under the SNMPv2 environment. The SNMPv2 agent also has a strong function (RowStatus SYNTAX ${ }^{(14)}$ ) on MIB table operation as well. If implementing the management system on SNMPV1, the sub manager must be embedded into the currently running SNMP agent. In this section, we describe how the management task is created through reading out the management policy saved in MPMIB. Two practical implementation examples are shown below.

5.1 Management Operations The sub manager always listens on the SNMP port to capture the SNMP packet (UDP type) coming in. Then, it expands the captured packet and analyzes to see if it is an Extended SNMP PDU for hierarchical network management. If the packet is a SetRequest / InformRequest PDU and XY is non-zero, this packet will be processed by a fork system call to write the management policy included in the SNMP PDU into the MPMIB. Otherwise, this packet is considered as a normal SNMP PDU packet from the manager to the agent and should be handed over to the SNMP agent on this sub manager. Another fork system call alerts the management task after the management policy is written into MPMIB and is ready to be read out. The management task creates GetRequest / GetNextRequest or SetRequest / InformRequest PDU by GETOPR (or GETNEXTOPR) / SETOPR operator to get/set the management information from/to the agents located in the sub network and then calculates the result the main manager wants. The operator that includes the arithmetic operations, the logical operations, and some other operations such as SQUARE, ROOT, and LOAD, WAIT, STORE controls the calculation and processing. The calculated result is stored into the MRMIB, which can then be accessed by the remote main manager via GetRequest / GetNextRequest / InformRequest PDU for retrieving result values or SetRequest PDU for deleting certain result values that are no longer considered valuable.

Some operators in common use are defined in Table 2. These operators help the main manager and sub manager achieve almost all of the purposes for their network management, and the set of operators can be enhanced and extended if necessary. As described in Section 4.1, the operand consists of a queue of ipAddress-object identifier pairs. The form of the ipAddress is defined in Table 3. Several special usages of ipAddress can improve the operability and the efficiency of management policy. For example, it is possible to set/get management information to/from various devices in the remote network by a single management policy by inserting a different ipAddress in the operand queue. For instance, xxx.xxx.xxx.255/xxx.xxx.0.0 indicates the all the managed objects in a certain broadcast domain or sub network domain. This sort of ipAddress depends on the practical network IP address, and it must work with RMON probes that discover and maintain all the address information of hosts in the remote network. In any case, 000.000 .000 .000 means the next coming string is not an object identifier, but a number string which is another kind of operand.

Table 2. Operator Definitions

\begin{tabular}{|c|c|c|}
\hline opCode & operator & Description \\
\hline$\# 00$ & GETOPR & Delivering GetRequest \\
\hline$\# 01$ & GETNEXTOPR & Delivering GetNextRequest \\
\hline \#02 & INFORMOPR & Delivering InformRequest \\
\hline$\$ 03$ & SETOPR & Delivering SetRequest \\
\hline$\# 04$ & TRAPOPR & Delivering Trap to main manager \\
\hline$\$ 10$ & $\mathrm{ADD}$ & Adding operand to a Variable \\
\hline$\# 11$ & SUB & Subtracting operand from a Variable \\
\hline$\# 12$ & MULT & Multiplying operand to a Variable \\
\hline$\# 13$ & DIV & Dividing operand into a Variable \\
\hline$\$ 20$ & SQUARE & SQUARE of a Variable \\
\hline$\# 21$ & ROOT & ROOT of a Variable \\
\hline$\# 30$ & AND & AND operand to a Variable \\
\hline$\# 31$ & $\mathrm{OR}$ & OR operand to a Variable \\
\hline$\# 32$ & XOR & XOR operand to Variabie \\
\hline$\# 40$ & JUDGESML & $\begin{array}{l}\text { If operand }<\text { Variable than Next step } \\
\text { else Next }+4 \text { step (ref. policy order) }\end{array}$ \\
\hline$\$ 41$ & JUDGELAG & $\begin{array}{l}\text { If operand }>\text { Variable than next step } \\
\text { else Next }+4 \text { step (ref. policy order) }\end{array}$ \\
\hline$\# 42$ & JUDGEEQU & $\begin{array}{l}\text { If operand=Variable than Next step } \\
\text { else Next }+4 \text { step (ref. policy order) }\end{array}$ \\
\hline$\# 43$ & SPACE & $\begin{array}{l}\text { Space step, counted as one program } \\
\text { step }\end{array}$ \\
\hline$\# 44$ & MARK & Indicating a entrance \\
\hline$\# 45$ & JUMPPREY & $\begin{array}{l}\text { Jump to previous MARK } \\
\text { (ref. policy order) }\end{array}$ \\
\hline$\# 46$ & JUMPNEXT & $\begin{array}{l}\text { Jump to next MARK } \\
\text { (ref. policy order) }\end{array}$ \\
\hline$\# 50$ & $\angle O A D$ & Moving operand to a Variable \\
\hline$\# 5$ & STORE & Storing a Variable to MRMIB \\
\hline$\# 52$ & WAIT & Waiting for Time (second) \\
\hline$\# 53$ & STOP & Halt and exit \\
\hline$\# \mathrm{~A} 0$ & Variableo & Variable $(0)$ \\
\hline \#A.I & Variablel & Variable(1) \\
\hline$:$ & & \\
\hline \#DF & VariableDF & Variable(63) \\
\hline
\end{tabular}

Table 3. Operand in Common Use (ipAddress part)

\begin{tabular}{|c|l|}
\hline ipAddress of operand & Description \\
\hline$x \times x \times x \times x . x \times x . x x x$ & IP address of an existing node \\
\hline$x \times x . x \times x . x x \times .255$ & To all nodes in a certain broadcast domain \\
\hline$x x x . x \times x .000 .000$ & To all nodes in a certain subnet domain \\
\hline 000.000 .000 .000 & $\begin{array}{l}\text { Indicating the upcoming data is an integer. } \\
\text { not an object identifier }\end{array}$ \\
\hline
\end{tabular}


5.2 Implementation Example Next, we present two implementation examples of our Hierarchical Network Management based on Extended SNMP.

The first example shows how to make a sub manager calculate the loss rate of packets received by an interface of a certain node (ipAddress $=X$ ). In practical network management, it is essential to obtain the information of packet loss rate in order to master the interface status of the network device, such as a host or a router. The packet loss rate can be described as the following expression (Fig. 6).

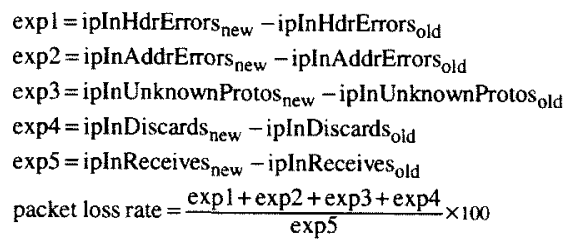

Fig. 6. Expression for Calculating the Packet Loss Rate

According to the expression shown in Fig. 6, the main manager creates the operator (management policy) and operand (managed object) shown in Fig. 7 and sends the opCode and operand to the sub manager's MPMIB by two SetRequest PDUs.

\begin{tabular}{|c|c|c|c|}
\hline \multicolumn{3}{|c|}{ To Operator Table of MPMIB } & \\
\hline opCode & operator & $l^{s}$ operand & $-2^{\text {nd }}$ operand \\
\hline$\# 01$ & \multicolumn{3}{|c|}{ GETNEXTOPR OperandTableRow } \\
\hline \#50A0 & LOAD & Variable0 & ipInHdrErrors (object value) \\
\hline$\# 10 \mathrm{AO}$ & $\mathrm{ADD}$ & Variableo & ipInAddrErrors (object value) \\
\hline \#10A0 & ADD & Variableo & ipInUnknownProtos (object value) \\
\hline$\# 10 \mathrm{AO}$ & ADD & Variableo & ipInDiscards (object value) \\
\hline$\# 50 \mathrm{Al}$ & LOAD & Variablel & ipInReceives (object value) \\
\hline$\# 52$ & WAIT & integerl $(=$ & time) \\
\hline$\# 01$ & \multicolumn{3}{|c|}{ GETNEXTOPR OperandTableRow } \\
\hline$\# 50 \mathrm{~A} 2$ & LOAD & Variable2 & ipInHdrErrors (object value) \\
\hline$\# 10 \mathrm{~A} 2$ & ADD & Variable2 & ipInAddrErrors (object value) \\
\hline$\# 10 \mathrm{~A} 2$ & ADD & Variable2 & ipInUnknownProtos (object value) \\
\hline$\# 10 \mathrm{~A} 2$ & ADD & Variable2 & ipInDiscards (object value) \\
\hline$\# 50 \mathrm{~A} 3$ & LOAD & Variable3 & ipInReceives (object value) \\
\hline \#11A2A0 & SUB & Variable2 & Variable 0 \\
\hline$\#|| A 3 A \mid$ & SUB & Variable3 & Variablel \\
\hline$\# 13 \mathrm{~A} 3 \mathrm{~A} 2$ & DIV & Variable3 & Variable2 \\
\hline$\# 12 \mathrm{~A} 3$ & MULT & Variable3 & integer $2(=100)$ \\
\hline$\# 04 \mathrm{A3}$ & TRAPOPR & Variable3 & \\
\hline$\# 51$ A3 & STORE & Variable3 & \\
\hline$\# 53$ & STOP & & \\
\hline
\end{tabular}

\section{To Operand Table of MPMIB}

ipAddressX ipInHdrErrors (object identifier $=" 1.3 .6 .1 .2 .1 .4 .4 "$ ) ipAddress $X$ ipInAddrErrors (object identifier $=" 1.3 .6 .1 .2 .1 .4 .5^{\prime \prime}$ ) ipAddress $X$ ipInUnknownProtos (object identifier ="1.3.6.1.2.1.4.7") ipAddress X ipInDiscards (object identifier $=" 1.3 .6 .1 .2 .1 .4 .8^{*}$ ) ipAddress $X$ ipInReceives (object identifier $=" 1 \cdot 3 \cdot 6 \cdot 1.2 .1 .4 .3 "$ ) non-ipAddress integer l (=time) non-jpAddress integer $2(=100)$

Fig. 7. Management Policy and its Operand (Example 1)

Then, the sub manager browses the MPMIB to create the Management Task. The GETNEXTOPR operator issues a
GetNextRequest PDU according to the IP address and object identifier from the Operand Table row entry. The replies from the managed agent are stored into a temporary FIFO data pipe and will be read out in turn from the LOAD operator. Each opCode also shows how to perform an operation. For example, \#11A2A0 means Variable2 Variable2-Variable0, and \#12A3 means Variable3 — Variable ${ }^{*}$ operand (next data coming from the FIFO pipe; it is 100 here). The final result is in Variable 3 and will be reported to the main manager by TRAPOPR and saved into the local MRMIB by STORE operator. Note that the retrieval key is the management policy index.

The second example (Fig. 8) shows how to monitor the interface's current bandwidth of specified 20 nodes (ipAddress $=A, B, \cdots, T$ ) in the remote network. The Trap PDU will be sent to the main manager if the estimate of any interface's bandwidth is lower than the specified threshold in advance.

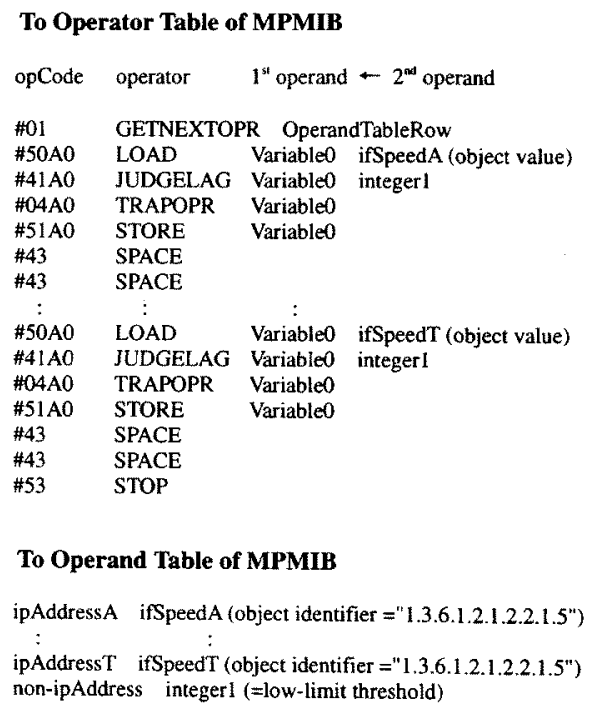

Fig. 8. Management Policy and its Operand (Example 2)

When to execute the above management policy, how long to execute it, and how many times can be decided by the main manager in advance only by setting the proper parameters into smExecStart, smExecInterval, and smExecTimes object. There were described in Sections 3.2 and 4.1. On the other hand, it is only necessary to send the other operand data to the sub manager, if the main manage would prefer to use the same management policy to get management information from any other network device located in the same remote network.

According to the results of above implementation examples, a simple function comparison table of several 
management methods is shown in following Table. 4. Using the normal SNMPv1/v2-based management method, the network manager has to setup a number of connections in order to collect basic object information from different managed nodes and needs CPU resource for calculating the high-level management results. The same management purpose can be achieved by the Hierarchical Network Management method we proposed, in which the amount of connection will be greatly reduced, and the main manager will require only a small load. In contrast, the same results for the given examples could not be achieved by the RMON method because of limited RMON MIB definitions.

Table 4. Management Function Comparison

\begin{tabular}{|c|c|c|c|c|}
\hline \multirow{2}{*}{$\begin{array}{l}\text { Function } \\
\text { Method }\end{array}$} & \multicolumn{2}{|c|}{ SNMP UDP Connection } & \multirow{2}{*}{$\begin{array}{l}\text { Main Manager } \\
\text { Calculation Load }\end{array}$} & \multirow{2}{*}{$\begin{array}{c}\text { High-level } \\
\text { Result }\end{array}$} \\
\hline & from Manager & to Manager & & \\
\hline SNMPv1v2 & $2 / 20$ & $2 / 20$ & $10 / 20$ & possible \\
\hline RMON & impossible & npossible & $0 / 0$ & Emited \\
\hline HNM & $2 / 2$ & $3 / 3$ & $0 / 0$ & possible \\
\hline
\end{tabular}

*cxämplel lexample2 "HNM: Hierarchical Network Management based on Extended SNMP

\section{Conclusions and Future Work}

In this paper, we presented a scheme of Hierarchical Network Management based on Extended SNMP to distribute most of management task to the sub manager. Firstly, the framework of hierarchical network management is defined. To implement this management framework on real networks, the SNMP PDU was developed and incorporated with the function to send the management policy to sub managers. Different management tasks are accomplished in local sub networks so that the load of the main manager and the relevant traffic on the backbone network could be decreased. The implementation examples showed how to use the free-designed management policies so as to realize the hierarchical network management on TCP/IP networks and obtain the high-level management results directly from a sub manager. The communications between the main manager and the sub manager are realized by the Extended SNMP which is an SNMP-based protocol so it is easy to construct a multi-level management structure. Certainly, the proposed method could help us to manage a large-scale network more easily and efficiently with the now popular SNMP, SNMPv2, or the upcoming SNMPv3. Depending on the proposed method and the platform, any network management software manufacturer could also develop more hierarchical management applications in accordance with its own management purposes.

Several other functions are also important for large-scale network management, such as the support method between one main manager and several sub managers. In the future work, we plan to develop this support method which can hand off the privilege of the main manager to a certain sub manager in order to manage the network continuously when the main manager fails to manage, or breaks down. These can enhance the reliability and fault tolerability of an entire hierarchical network management system. Furthermore, we will propose our solution to the IETF Working Group and expect it to become an additional standard of the InternetStandard Management Framework so as to keep up with the trend of networks becoming increasingly distributed, geographically and logically.

(Manuscript received July 29, 1998; revised Oct. 26, 1998)

\section{References}

(1) A. Leinwamd, K. Fang, Network Management-A Practical Perspective, Addison-Wesley Inc., 1993.

(2) J. Case, K. McCloghrie, M. Rose, S. Waldbusser, Manager-toManager Management Information Base (RFC1451), IETF, April 1993.

(3) G. Held, LAN Management with SNMP and RMON, John Wiley \& Sons Inc., 1996.

(4) H. Ogane, Network Management for TCP/IP and OSI, SRC, 1993.

(5) W. Stallings, SNMP, SNMPy2, and CMIP: the Practical Guide to Network Management Standards, Addison-Wesley Inc., 1993.

(6) M.A. Miller, Managing Internetworks with SNMP, $2^{\text {nd }}$ Edition, M\&T Books, A Division of MIS: Press Inc., 1997.

(7) SNMPv3 White Paper - Introduction to SNMPv3, SNMP Research International Inc., April 1998

(8) J. Case, M. Fedor, M.L. Schoffstall, C. Davin, Simple Network Management Protocol (SNMP) (RFC1157), IETF, May 1990.

(9) J. Case, K. McCloghrie, M. Rose, S. Waldbusser, Protocol Operations for version 2 of the Simple Network Management Protocol (SNMPV2) (RFC1448), IETF, April 1993.

(10) J. Case, K. McCloghrie, M. Rose, S. Waldbusser, Coexistence between Version 1 and Version 2 of the Internet-standard Network Management Framework (RFC1908), IETF, January 1996.

(11) D. Levi, P. Meyer, B. Stewart, SNMPv3 Applications (RFC2273), IETF, January 1998.

(12) CCITT X.409, ASN.1 (Abstract Syntax Notation One), 1984.

(13) M. Rose, K. McCloghrie, Concise MIB Definitions (RFC1212), IETF, March 1991 .

(14) J. Case, K. McCloghrie, M. Rose, S. Waldbusser, Textual Conventions for Version 2 of the Simple Network Management Protocol (SNMPv2) (RFC1443), IETF, April 1993.

(15) M. Rose, An Introduction to Management of TCP/IP based on Internets, Prentice-Hall Inc., 1991. 
Yu Guo (student member) received his B.E. degree from East

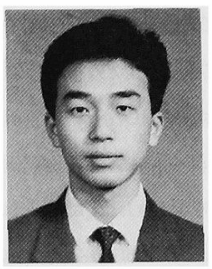

China Normal University, Shanghai, China in 1990 and received his M.E. degree from Yamagata University, Yonezawa, Japan in 1996. He was an Assistant Engineer in the department of Automatic Control, Jiao Tong University, Shanghai, China from 1990 to 1992 . He is now a Ph.D. candidate at the Engineering Faculty of Yamagata University. His research interests include large-scale network management and autonomic communication buffer control.

Yukio Hiranaka (non-member) received his B.E., M.E., and

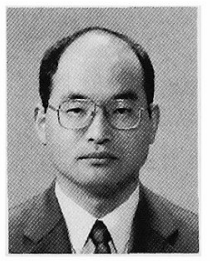
$\mathrm{Ph} . \mathrm{D}$. from the University of Tokyo, in 1976, 1978 and 1988, respectively, all in Information Physics. He was a Research Associate of the Department of Mathematical Engineering and Information Physics, University of Tokyo, from 1980 to 1989 . He is presently an Associate Professor of Electrical and Information Engineering of the Faculty of Engineering, Yamagata University, Yonezawa, Japan. He has worked on multi-dimensional intelligent sensing technology and real-time visualization of sound and smell. Now, his interests include general computer networking technologies as well.

Takao Akatsuka (non-member) received his B.E., M.E., and

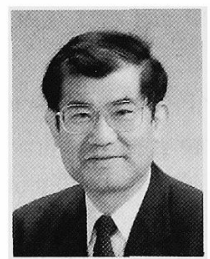
Ph.D. from the University of Tokyo, in 1964, 1966 and 1974, respectively. From 1969 to 1974 , he was a Research Associate of the Department of Mathematical Engineering and Information Physics, University of Tokyo, and from 1974 to 1983, he was Assistant Professor of Medical Engineering at the University of Tsukuba from 1984. He is a Professor of Electrical and Information Engineering of the Faculty of Engineering, Yamagata University, Yonezawa, Japan His interests are imaging technology and image handling in computer networks. 